\title{
Tromboprofilassi nel paziente con sindrome di Cushing
}

\author{
Silvia Pinelli ${ }^{1}$. Carla Scaroni ${ }^{1}$ - Filippo Ceccato ${ }^{1}$. Mattia Barbot ${ }^{1}$
}

Accettato: 7 aprile 2020 / Pubblicato online: 27 maggio 2021

(c) The Author(s) 2021

\section{Introduzione}

La sindrome di Cushing è una condizione clinica conseguente all'esposizione prolungata a livelli eccessivi di glucocorticoidi circolanti. La causa più frequente è quella iatrogena, che si sviluppa nel contesto di patologie che comportino l'assunzione cronica di corticosteroidi a scopo terapeutico. La forma endogena è una malattia rara, caratterizzata da un'iperproduzione di glucocorticoidi da parte dei surreni, a sua volta distinta in una forma $\mathrm{ACTH}$-dipendente e in una ACTH-indipendente.

Numerosi studi hanno evidenziato, tra i pazienti con sindrome di Cushing, l'aumento del rischio di eventi tromboembolici sia postoperatori che spontanei, che contribuiscono a incrementare la morbidità e la mortalità cardiovascolare di questi pazienti [1].

\section{Rischio di eventi trombo-embolici spontanei}

Tutti i fattori della triade di Virchow (ipercoagulabilità, alterazioni emodinamiche e disfunzione endoteliale) sono implicati nella patogenesi del trombo-embolismo nella sindrome di Cushing:

- ipercoagulabilità: è legata all'aumento dei livelli di alcuni fattori della coagulazione, in particolare fattore VIII (FVIII) e fattore di von Willebrand (vWF), e a un'alterata capacità fibrinolitica;

$\triangle$ F. Ceccato

filippo.ceccato@unipd.it

1 U.O. Endocrinologia, Dipartimento di Medicina DIMED, Università di Padova, Azienda Ospedaliero-Università di Padova, Padova, Italia
- alterazioni emodinamiche: il flusso sanguigno è alterato a causa di policitemia, obesità, ridotta mobilità;

- disfunzione endoteliale: il cortisolo causa rimodellamento concentrico cardiaco e ispessimento delle pareti arteriolari, tramite l'attivazione del recettore dei mineralcorticoidi. Anche la regolazione della vasodilatazione viene meno e numerosi marker di disfunzione endoteliale (endotelina, VEGF, omocisteina) risultano patologici [2].

Inoltre, la sindrome di Cushing spesso si associa ad altri fattori di rischio (obesità, diabete, ipertensione, dislipidemia, infezioni, immobilizzazione da danno osteoporotico vertebrale), che aggravano l'ipercoagulabilità secondaria all'ipercorticismo endogeno [3].

Uno studio condotto su 176 pazienti seguiti presso il nostro Centro ha identificato alcuni parametri che sembrano correlati a un maggiore rischio trombo-embolico: l'età $\geq 69$ anni, le infezioni, la ridotta mobilità, il cortisolo salivare notturno $>3,15 \mathrm{ULN}$, la riduzione del tempo di tromboplastina parziale attivata (aPTT), precedenti cardiovascolari [4].

Nonostante tali evidenze, non esiste ad oggi uno score condiviso di valutazione del rischio che determini le condizioni in cui sia indicata una profilassi antitrombotica durante le fasi di ipercorticismo attivo e il rapporto rischio/beneficio deve essere valutato per ogni singolo caso.

\section{Rischio di eventi trombo-embolici correlati alla chirurgia}

Particolare attenzione merita il rischio trombo-embolico correlato alla chirurgia, che rappresenta l'opzione terapeutica di prima scelta per tutte le forme di sindrome di Cushing. L'intervento chirurgico può infatti alterare il delicato equilibrio coagulativo, favorendo uno stato pro-infiammatorio e pro-coagulante probabilmente legato alla rapida riduzione 
dei livelli ormonali, con ulteriore aumento di FVIII e vWF e incremento del rischio trombotico [3].

Uno studio retrospettivo condotto nel 2011 su 473 pazienti ha evidenziato una maggiore incidenza di eventi trombo-embolici tra i pazienti sottoposti a chirurgia ipofisaria per malattia di Cushing rispetto a quelli sottoposti allo stesso intervento per adenoma ipofisario non secernente [1]. Il ripristino dei normali valori dei parametri coagulativi dopo l'intervento può richiedere mesi [5]; un recente studio ha riscontrato come il rischio trombo-embolico sia più elevato nei 60 giorni che seguono la chirurgia ipofisaria e la bisurrenectomia, senza correlazione tra profilassi antitrombotica e aumento del rischio di sanguinamento o di complicanze chirurgiche [6].

Emerge, quindi, l'utilità di una profilassi antitrombotica peri-chirurgica per i pazienti che non presentino diatesi emorragica, seppure non esistano linee guida riguardo la tipologia e la durata di tale trattamento. Sulla base di uno studio retrospettivo condotto sulla nostra casistica e coinvolgente 78 pazienti, riteniamo che la strategia più sicura ed efficace in caso di chirurgia ipofisaria trans-sfenoidale per malattia di Cushing sia la seguente:

- favorire la mobilizzazione precoce, a partire dal secondo giorno dopo l'intervento;

- far indossare al paziente calze elastiche dal giorno del ricovero fino a completa mobilizzazione;

- somministrare eparina a basso peso molecolare (EPBM) 4.000 UI s.c./die per 30 giorni, a partire da 24 ore dopo l'intervento. In caso di pazienti di peso superiore a $80 \mathrm{~kg}$, il dosaggio consigliato è 8.000 UI sc die [3].

\section{Conclusioni}

Il trombo-embolismo è una complicanza importante della sindrome di Cushing. Per una corretta stratificazione del rischio pro-trombotico in questi pazienti è necessario valutare i fattori di rischio classici concomitanti e i parametri laboratoristici coagulativi. La profilassi antitrombotica nel periodo postoperatorio è efficace e sicura in assenza di concomitante diatesi emorragica. Durante le fasi attive di malattia, in cui si evidenzino situazioni a rischio trombotico (infezioni, immobilizzazione), la terapia con eparina a basso peso molecolare potrebbe essere utile, ma va valutata caso per caso in base ai fattori di rischio.
Funding Note Open access funding provided by Università degli Studi di Padova within the CRUI-CARE Agreement.

Conflitto di interesse Gli autori Silvia Pinelli, Carla Scaroni, Filippo Ceccato e Mattia Barbot dichiarano di non avere conflitti di interesse.

Consenso informato Lo studio presentato in questo articolo non ha richiesto sperimentazione umana.

Studi sugli animali Gli autori di questo articolo non hanno eseguito studi sugli animali.

Nota della casa editrice Springer Nature rimane neutrale in riguardo alle rivendicazioni giurisdizionali nelle mappe pubblicate e nelle affiliazioni istituzionali.

Open Access This article is licensed under a Creative Commons Attribution 4.0 International License, which permits use, sharing, adaptation, distribution and reproduction in any medium or format, as long as you give appropriate credit to the original author(s) and the source, provide a link to the Creative Commons licence, and indicate if changes were made. The images or other third party material in this article are included in the article's Creative Commons licence, unless indicated otherwise in a credit line to the material. If material is not included in the article's Creative Commons licence and your intended use is not permitted by statutory regulation or exceeds the permitted use, you will need to obtain permission directly from the copyright holder. To view a copy of this licence, visit http://creativecommons.org/licenses/by/4.0/.

\section{Bibliografia}

1. Stuijver DJ, van Zaane B, Feelders RA et al (2011) Incidence of venous thromboembolism in patients with Cushing's syndrome: a multicenter cohort study. J Clin Endocrinol Metab 96(11):3525-3532

2. Miljic P, Miljic D, Cain JW et al (2012) Pathogenesis of vascular complications in Cushing's syndrome. Horm Athens Greece 11(1):21-30

3. Barbot M, Daidone V, Zilio M et al (2015) Perioperative thromboprophylaxis in Cushing's disease: what we did and what we are doing? Pituitary 18(4):487-493

4. Zilio M, Mazzai L, Sartori MT et al (2016) A venous thromboembolism risk assessment model for patients with Cushing's syndrome. Endocrine 52(2):322-332

5. Manetti L, Bogazzi F, Giovannetti C et al (2010) Changes in coagulation indexes and occurrence of venous thromboembolism in patients with Cushing's syndrome: results from a prospective study before and after surgery. Eur J Endocrinol 163(5):783-791

6. Suarez MG, Stack M, Hinojosa-Amaya JM et al (2020) Hypercoagulability in Cushing syndrome, prevalence of thrombotic events: a large, single-center, retrospective study. J Endocr Soc 4(2):bvz033 